OPEN ACCESS

Edited by:

Katrin Kahlen,

Hochschule Geisenheim University,

Germany

Reviewed by:

Norihito Nakamichi,

Nagoya University, Japan

Matt Jones,

University of Essex, United Kingdom

*Correspondence:

Takayuki Ohara

t.ohara.63920@ees.hokudai.ac.jp

Specialty section:

This article was submitted to Plant Biophysics and Modeling,

a section of the journal

Frontiers in Plant Science

Received: 31 August 2017 Accepted: 11 October 2017 Published: 30 October 2017

Citation:

Ohara T and Satake A (2017)

Photosynthetic Entrainment of the

Circadian Clock Facilitates Plant

Growth under Environmental

Fluctuations: Perspectives from an Integrated Model of Phase Oscillator and Phloem Transportation.

Front. Plant Sci. 8:1859. doi: 10.3389/fp/s.2017.01859

\section{Photosynthetic Entrainment of the Circadian Clock Facilitates Plant Growth under Environmental Fluctuations: Perspectives from an Integrated Model of Phase Oscillator and Phloem Transportation}

\author{
Takayuki Ohara ${ }^{1,2 *}$ and Akiko Satake ${ }^{2}$ \\ ${ }^{1}$ Graduate School of Environmental Science, Hokkaido University, Sapporo, Japan, ${ }^{2}$ Department of Biology, Faculty of \\ Science, Kyushu University, Fukuoka, Japan
}

Plants need to avoid carbon starvation and resultant growth inhibition under fluctuating light environments to ensure optimal growth and reproduction. As diel patterns of carbon metabolism are influenced by the circadian clock, appropriate regulation of the clock is essential for plants to properly manage their carbon resources. For proper adjustment of the circadian phase, higher plants utilize environmental signals such as light or temperature and metabolic signals such as photosynthetic products; the importance of the latter as phase regulators has been recently elucidated. A mutant of Arabidopsis thaliana that is deficient in phase response to sugar has been shown, under fluctuating light conditions, to be unable to adjust starch turnover and to realize carbon homeostasis. Whereas, the effects of light entrainment on growth and survival of higher plants are well studied, the impact of phase regulation by sugar remains unknown. Here we show that endogenous sugar entrainment facilitates plant growth. We integrated two mathematical models, one describing the dynamics of carbon metabolism in $A$. thaliana source leaves and the other growth of sink tissues dependent on sucrose translocation from the source. The integrated model predicted that sugar-sensitive plants grow faster than sugar-insensitive plants under constant as well as changing photoperiod conditions. We found that sugar entrainment enables efficient carbon investment for growth by stabilizing sucrose supply to sink tissues. Our results highlight the importance of clock entrainment by both exogenous and endogenous signals for optimizing growth and increasing fitness.

Keywords: starch degradation, sucrose, homeostasis, mathematical model, dynamics, phloem transportation, Arabidopsis thaliana

\section{INTRODUCTION}

Plants are inevitably exposed to daily and seasonal variations in light environments. To continuously grow in fluctuating environments, it is crucial for plants to stably supply carbon resources for respiration and growth. Plant growth in the day is supported by the supply of photosynthates, particularly soluble sugars such as sucrose that are transported from 
photosynthetic leaves (source tissues) to sink tissues (e.g., roots). Plants grow even in nighttime using carbon resources accumulated during the preceding daytime. Arabidopsis thaliana, a model plant, partitions a large fraction of assimilated carbon into insoluble starch, which is degraded at night to produce sucrose (Smith and Stitt, 2007; Stitt and Zeeman, 2012). Because early exhaustion of starch results in carbon starvation and ensuing growth inhibition (Graf et al., 2010; Yazdanbakhsh et al., 2011), careful management of starch metabolism is essential to cope with daily and seasonal fluctuations of light conditions.

In $A$. thaliana, starch amount increases during the day at an almost constant rate and decreases almost linearly at night (Caspar et al., 1985; Gibon et al., 2004; Smith et al., 2004; Lu et al., 2005). Plants in shorter photoperiods accumulate starch more rapidly during the day and degrade it more slowly at night than in longer photoperiods (Lu et al., 2005). Plants also adjust the rate of starch degradation immediately in response to an unexpectedly early or late onset of night (Lu et al., 2005; Graf et al., 2010; Scialdone et al., 2013). The circadian clock underlying the approximately 24 -h cycle of biological processes is implicated in the control of starch metabolism (Graf and Smith, 2011). Wild type A. thaliana (Ws) exhausts starch reserves about $24 \mathrm{~h}$ after the last dawn even under non-24 h light/dark cycles (T-cycles) (Graf et al., 2010), indicating that the timing of starch exhaustion is programmed by the circadian clock. In the circadian clock mutant ccal/lhy, in which the functional clock has a period of about $17 \mathrm{~h}$ (Locke et al., 2005), the depletion of starch occurs prematurely under a 24 -h T-cycle but coincides with dawn under a 17-h T-cycle (Graf et al., 2010). These studies suggest that coordination of the internal timing of starch turnover with environmental cycles is necessary to avoid carbon starvation.

Phase adjustment of the circadian clock to external stimuli such as light or temperature is fundamental for synchronizing biological processes with environments (Johnson et al., 2003). In addition to signals from the external environment, endogenous signals such as photosynthates are also important regulators of the circadian phase in A. thaliana. We previously reported that phase adjustment by sugar is necessary for plants to flexibly regulate carbon metabolism in fluctuating light environments (Seki et al., 2017). We developed a phase oscillator model describing phase regulation of the circadian clock by sucrose. This model predicted that phase adjustment of the circadian clock by sucrose is crucial for homeostatic regulation of carbon resources. These theoretical predictions were confirmed by physiological experiments using the mutant pseudoresponse regulator 7-11 (prr7-11), the circadian clock of which does not show clear phase response to sucrose pulse (Haydon et al., 2013).

Whereas, clock entrainment by exogenous signals such as light has been shown to be advantageous for competition and survival in several organisms (Woelfle et al., 2004; Dodd et al., 2005), the advantages of clock entrainment by photosynthetic products remain elusive. Here we theoretically evaluate the effects of clock entrainment by sugar on plant growth, a good proxy for plant fitness (Younginger et al., 2017). We extended the phase oscillator model for the circadian clock (Seki et al., 2017) by incorporating growth dynamics of sink tissues, including shoot apical meristems and roots. Growth dynamics are described by modeling phloem transportation of sucrose from source to sink tissues (Seki et al., 2015; Satake et al., 2016). We demonstrate that plant growth is facilitated by endogenous sugar entrainment under long photoperiods because the entrainment enables the stable supply of sucrose from source to sink tissues irrespective of light fluctuation. In short photoperiods, however, the effect of sugar entrainment on growth is negligibly small. Our results provide important theoretical evidence that circadian-phase adjustment by endogenous signals is advantageous for plant growth.

\section{MODEL AND METHODS}

To investigate the effect of clock regulation by sugar on plant growth, we integrated two previously developed models, one describing the dynamics of starch and sucrose metabolism in source leaves (Seki et al., 2017) and the other growth of sink tissues dependent on phloem transportation of sucrose from source tissues (Seki et al., 2015; Satake et al., 2016). Photosynthetic products in the source leaf are partitioned into sucrose and starch (Seki et al., 2017; Figure 1A). Sucrose in the source leaf is loaded into the phloem, moves through the phloem tube, and is unloaded at sink tissues where it is used for respiration and growth (Seki et al., 2015; Satake et al., 2016; Figures 1B-D). These two models were coupled by incorporating a term that represents sucrose translocation from source to sink. We explain the detailed structures of each component of our new integrated model in the following sections. We consider two sink tissues, the shoot and root apical meristems (SAM and RAM, respectively), because plant growth and development mainly occur in these organs (Figure 1C). Our model can be extended to the structure including multiple sinks in the complex phloem network as studied previously (Seki et al., 2015; Satake et al., 2016).

\section{Sugar Dynamics in Source Leaves and Sink Tissues Source Leaves}

In source leaves (Figure 1A), carbon is assimilated by photosynthesis at a rate $a$ during the light period. The length of the light period is given by $\tau_{\mathrm{L}}$. A fraction $\gamma$ of total photoassimilates is partitioned into starch $(C)$ for storage and a fraction $1-\gamma$ is partitioned into sucrose $\left(S_{\mathrm{G}}\right)$. Sucrose is consumed for respiration at a rate $h_{\mathrm{G}}$ and for transportation at a rate $\eta_{G}$. Starch is degraded into sucrose at a rate $\beta$, which is assumed to be under the control of the circadian clock and thus is a function of the phase $\phi$ of the circadian oscillator (Seki et al., 2017). These processes are formalized as follows:

$$
\begin{aligned}
\frac{d}{d t} S_{G}(t) & =a L(t)(1-\gamma)+\beta(\phi) C^{\kappa}-\left(h_{G}+\eta_{G}\right) S_{G}, \\
\frac{d}{d t} C(t) & =a L(t) \gamma-\beta(\phi) C^{\kappa},
\end{aligned}
$$

where $L(t)$ indicates the light condition (defined as 1 under light and 0 under dark) and $\kappa$ is a constant. Starch reserve is accumulated during the light period at a rate determined by 
A

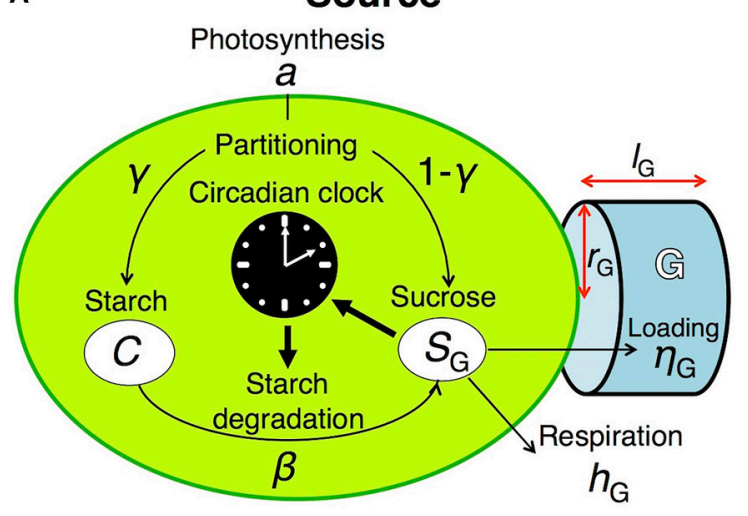

Source leaf Phloem tube

c

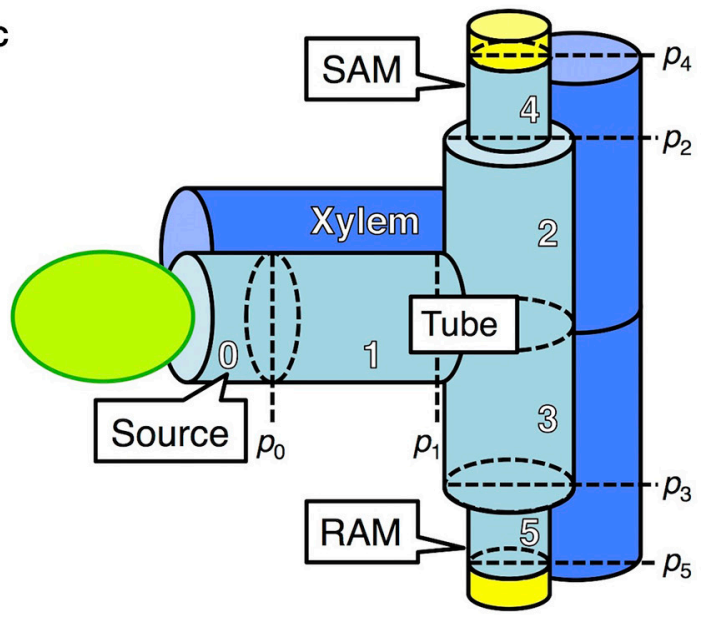

B

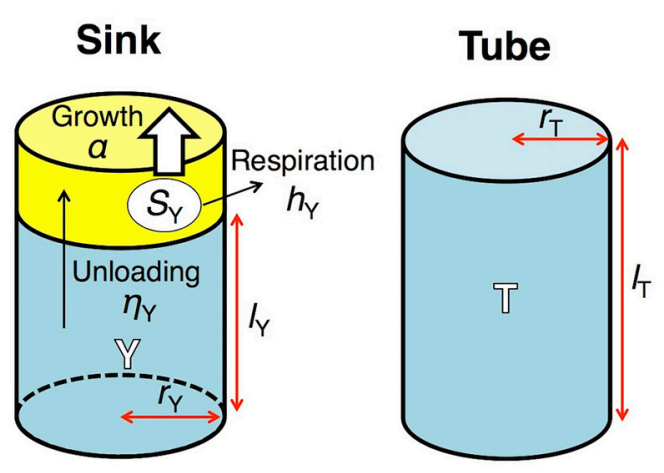

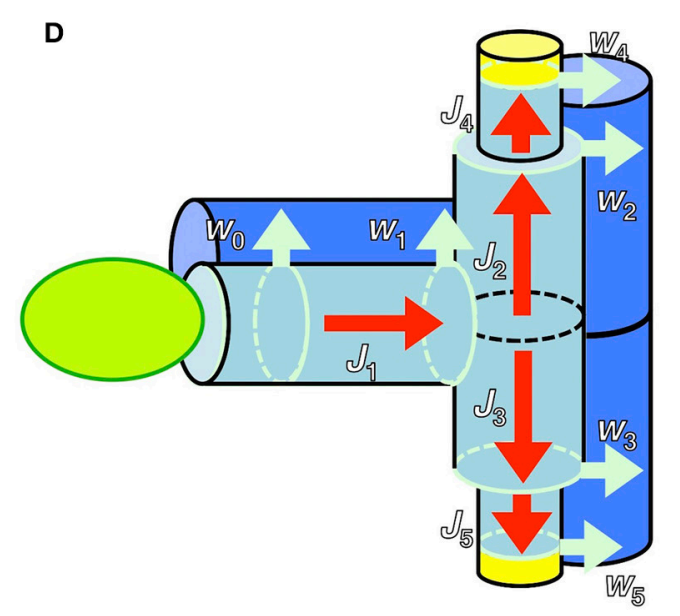

FIGURE 1 | Model describing sugar dynamics in source and sink tissues and translocation of sucrose through the phloem tube. (A) In a source leaf, the circadian clock and carbon metabolism are reciprocally regulated. (B) Translocated sucrose is used for growth and respiration in the sink. Each structural component is expressed by a cylinder of radius $r_{X}$ and height $/ X$, where $X$ is either $G$ (source), $T$ (tube), or $Y$ (sink). (C) Tubal structure of the model. $p_{i}$ represents hydrostatic pressure at the apex of component $i(i \in\{0, \ldots, 5\})$. Xylem adjacent to the phloem tube is also schematized. SAM, shoot apical meristem; RAM, root apical meristem. (D) Fluxes in the model. Red arrows $\left(J_{i}\right)$ indicate phloem sap flow. Light blue arrows $\left(w_{i}\right)$ indicate pure water flow due to osmosis, which occurs at the region represented by light blue circles.

balance between $a \gamma$ and $\beta C^{\kappa}$. The starch degradation rate $\beta(\phi)$ is assumed to show diel oscillation due to regulation by the circadian clock. We assume that $\beta(\phi)$ shows a peak at dawn and a trough at the subjective dusk $\phi^{*}$ because a previous study showed that this oscillation pattern is ideal to minimize fluctuations in the sucrose supply to sinks (Figure S1A; Seki et al., 2017).

In the previous model (Seki et al., 2017), transportation of sucrose from source leaves was assumed to be constant. In our new model, sucrose transportation is assumed to occur based on the pressure-flow hypothesis (Münch, 1930) with an assumption that the flux in phloem obeys the Hagen-Poiseuille law (Supplementary Material, section 1). Sucrose dynamics are now described both in the source leaves and phloem tubes. At the source leaves, sucrose is loaded into the adjacent phloem tube at a rate $\eta_{\mathrm{G}}$ (Figure 1A). At the phloem tube, loaded sucrose is transported to sink tissues (see Supplementary Material, section 1 for detailed explanation). The dynamics of sucrose concentration at the phloem tube adjacent to the source $\left(g_{0}\right)$ is given by

$\frac{d}{d t} g_{0}(t)=\frac{1}{V_{0}}\left\{\eta_{G} S_{G}(t)+g_{1}(t)\left[-J_{1}(t)\right]_{+}-g_{0}(t)\left[J_{1}(t)\right]_{+}\right\},(3)$ where $g_{1}(t)\left[-J_{1}(t)\right]_{+}-g_{0}(t)\left[J_{1}(t)\right]_{+}$describes the rate of sucrose change due to flux $J_{1}(t)$ at phloem tube 1 (Figures 1C,D). $g_{1}$ and $V_{0}$ represent sucrose concentration at tube 1 and the volume of tube 0 , respectively (Figure 1C).

Similar to the previous model (Seki et al., 2017), the phase $\phi$ of the circadian oscillator is modeled by

$$
\frac{d}{d t} \phi(t)=\omega+Z_{L}(\phi) f_{L}(\tilde{L})+Z_{S}(\phi) f_{S}\left(\tilde{S}_{G}\right)
$$

where $\omega$ is the angular frequency of the oscillator. The term $Z_{\mathrm{L}}(\phi) f_{\mathrm{L}}(\tilde{L})$ represents the effect of light stimulus, which is 
assumed to reset the phase to 0 at dawn and to $\tau_{\mathrm{L}}$ at dusk (Seki et al., 2017). $Z_{S}(\phi)$ is a phase response curve (PRC) to a sucrose pulse showing phase advance until $\phi=\phi^{*}$ and phase delay thereafter as previously determined (Figure S1B; Seki et al., 2017). Sugar input $f_{S}$ (Supplementary Material, section 2) is defined by the Hill function of the rate of change in sucrose level (i.e., $\tilde{S}_{G}=d S_{G} / d t$ ). The functions of $Z_{S}(\phi)$ and $f_{S}$ have been demonstrated to be optimal for minimizing sucrose fluctuation (Seki et al., 2017). We assume that the phase shift of the circadian clock by sugar takes place only in the light period because the phase shift at night did not improve sucrose homeostasis (Seki et al., 2017).

\section{Sink Tissues}

Translocated sucrose is unloaded into the sinks at a rate $\eta_{\mathrm{Y}}$ from the adjacent phloem tubes with sucrose concentration $g_{i}$ (Figure 1B). Sucrose in the $\operatorname{sink}\left(S_{\mathrm{Y}}\right)$ is consumed for respiration and growth at rates $h_{\mathrm{Y}}$ and $\alpha\left(S_{\mathrm{Y}}\right)$, respectively. These processes are formalized as follows:

$$
\begin{aligned}
\frac{d}{d t} g_{i}(t) & =\frac{1}{V_{i}}\left\{g_{i-2}(t)\left[J_{i}(t)\right]_{+}-g_{i}(t)\left(\left[-J_{i}(t)\right]_{+}+\eta_{\mathrm{Y}}\right)\right\} \\
\frac{d}{d t} S_{\mathrm{Y}}(t) & =\eta_{\mathrm{Y}} g_{i}(t)-h_{\mathrm{Y}} S_{\mathrm{Y}}(t)-\alpha\left(S_{\mathrm{Y}}\right)
\end{aligned}
$$

where $V_{i}$ is the volume of component $i$ ( $i=4$ for SAM and $i=5$ for RAM; $i=1,2$, and 3 for connecting tubes between the source and sink; Figure 1C). The first and second terms in the right-hand side of Equation (5) represent the solution inflow and outflow, respectively (see Supplementary Material, section $1)$. The function for sucrose consumption rate for growth $\alpha\left(S_{Y}\right)$ will be explained in the next subsection. To clarify the effect of sugar entrainment on plant growth, we simplified the model by assuming that the sugar and growth dynamics of SAM and RAM are identical. Therefore, the values of $\eta_{\mathrm{Y}}$ and $h_{\mathrm{Y}}$ as well as the parameters in $\alpha\left(S_{\mathrm{Y}}\right)$ are the same in the two sinks.

\section{Growth of Sink Tissues Growth Dynamics}

We formalized the growth kinetics of the sink tissue based on sucrose supply because a strong correlation between growth rate and sucrose supply has been reported in both the light and dark periods for A. thaliana (Sulpice et al., 2014; Mengin et al., 2017). When sugar supply is sufficient, growth is promoted by the target of rapamycin (TOR) kinase, the expression level of which correlates with $A$. thaliana shoot and root growth (Deprost et al., 2007; Lastdrager et al., 2014). On the contrary, Snf1-related kinase 1 (SnRK1) inhibits growth in response to low carbon availability (Baena-González and Sheen, 2008; Lastdrager et al., 2014). In addition, growth rate is likely to be saturated as sucrose supply is increased (Sulpice et al., 2014). Given these empirical findings, we assume that growth-related sucrose consumption rate is an increasing and saturating function of sucrose supply (Figure 2A):

$$
\alpha\left(S_{\mathrm{Y}}\right)=\alpha_{\operatorname{Max}} \frac{S_{\mathrm{Y}}^{\tilde{n}}}{\tilde{K}^{\tilde{n}}+S_{\mathrm{Y}}^{\tilde{n}}},
$$

where $\alpha_{\text {Max }}, \tilde{n}$, and $\tilde{K}$ are constants. These values are estimated using the published data of fresh biomass in A. thaliana (Caspar et al., 1985) as explained later. The rate of increase in sink fresh biomass $\left(W_{\mathrm{Y}}\right)$ is then described by

$$
\frac{d}{d t} W_{\mathrm{Y}}(t)=\lambda \alpha\left(S_{\mathrm{Y}}\right) W_{\mathrm{Y}}(t),
$$

where $\lambda$ is the conversion rate of sucrose for growth. Therefore, growth rate is represented by $\lambda \alpha\left(S_{\mathrm{Y}}\right)$. Since dry weight and fresh weight of $A$. thaliana Col-0 display qualitatively similar increase patterns (Caspar et al., 1985; Christophe et al., 2008), our model can also be applicable to the analysis of dry biomass by appropriate scaling. We tested the robustness of our results by using an alternative formalization of growth rate as a linear increasing function of sucrose (Supplementary Material, section 3; Figures S4, S5).

\section{Parameter Estimation of the Sucrose Consumption Rate for Growth}

The parameter values of the sucrose consumption rate $\alpha\left(S_{\mathrm{Y}}\right)$ in Equation (7) were estimated by fitting the simulated growth curves to published data measuring fresh weight of $A$. thaliana wild type (Col-0) and the starchless phosphoglucomutase (pgm)
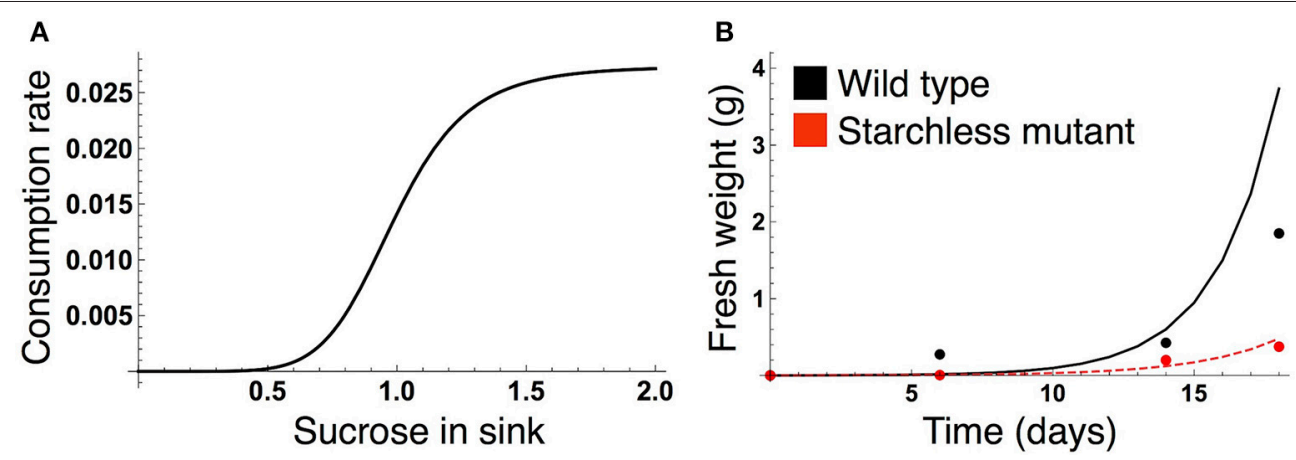

FIGURE 2 | (A) Sucrose consumption rate $\alpha$ for growth of the sink (SAM or RAM). The unit for sucrose is $\mu \mathrm{molC}_{6} \mathrm{~g}^{-1} \mathrm{FW}$. (B) Time evolution of growth of the wild type (black) and the starchless pgm mutant (red) grown in a 12-h photoperiod. In (B), lines represent the sum of the simulated growth of the two sinks and circles represent the published growth data (Caspar et al., 1985). 
mutant grown in a 12-h photoperiod (Caspar et al., 1985; Figure 2B). Because the number of the data is limited (data at four time points per genotype), we combine the data of both genotypes for the parameter estimation. The subjective dusk $\phi^{*}$ was set to $12 \mathrm{~h}$ because both the wild type and $p g m$ were likely to be completely entrained to a 12 -h photoperiod in the experiment. We simulated the growth of the wild type (see next subsection) and starchless mutant using an initial value of 0.0005 at time $t=$ 0 (corresponding to the first observation of plant fresh weight in the experiment). Because the pgm mutant does not accumulate starch, the carbon partitioning rate for starch ( $\gamma$ in Equations 1 and 2) was set to 0 for the mutant. The parameter values $\alpha_{\operatorname{Max}}, \tilde{n}$, and $\tilde{K}$ in Equation (7) were estimated by minimizing a following cost function:

$$
P\left(\alpha_{\mathrm{Max}}, \tilde{n}, \tilde{K}\right)=\sum_{X} \sum_{i=1}^{4}\left\{W_{i}^{E X P}-2 W_{Y, i}\left(\alpha_{\mathrm{Max}}, \tilde{n}, \tilde{K}\right)\right\}^{2}
$$

where $W_{i}^{E X P}$ and $W_{\mathrm{Y}, i}$ are fresh weight at the $i$ th time point in the experiment and the growth at corresponding times in the simulation, respectively (Figure 2B). $X$ corresponds to the wild type and pgm mutant. As fresh weight is likely to be measured from whole plants in the experiment (Caspar et al., 1985), we used the sum $\left(2 W_{\mathrm{Y}, i}\right)$ of the growth of SAM $\left(W_{\mathrm{Y}, i}\right)$ and RAM (also $W_{\mathrm{Y}, i}$ ) for the parameter estimation.

\section{Simulation Conditions}

To evaluate the effect of clock entrainment by sugar on growth, we simulated the growth dynamics of sugar-sensitive (wild type) and sugar-insensitive (mutant) plants in constant photoperiods (ranging from 8 to $16 \mathrm{~h}$ ) as well as under changing photoperiod conditions. The wild type adjusts the phase of the circadian clock by sugar as formalized in Equation (4), while the sugarinsensitive mutant lacks this response to sugar [i.e., $f_{\mathrm{S}}\left(\tilde{S}_{\mathrm{G}}\right)$ in Equation (4) is zero]. Both plants respond to light signals in the same manner. Although phase regulation by sugar reduces fluctuation of carbon resources, sucrose dynamics still deviate from homeostasis in both the wild type and mutant unless these plants precisely predict the timing of dusk (i.e., $\phi^{*}=\tau_{\mathrm{L}}$; Seki et al., 2017). To investigate the effect of sucrose homeostasis on growth, we also consider an ideal plant that can maintain perfect homeostasis in a steady state in any photoperiod (hereafter termed the homeostatic plant). We previously determined the optimal function for starch degradation rate to minimize sucrose fluctuation in a given photoperiod (Seki et al., 2017). The homeostatic plant is assumed to possess these functions in each photoperiod and is simulated by setting $\phi^{*}$ being equal to $\tau_{\mathrm{L}}$ in any condition $\left(\tau_{\mathrm{L}} \epsilon(0,24]\right)$ in contrast to the fixed value of $\phi^{*}$ in the wild type and sugar-insensitive mutant (summarized in a table in Figure S1). Comparison of growth dynamics among these three plants enables us to address the differential effects of endogenous sugar entrainment and sucrose homeostasis on growth. Because SAM and RAM grow at similar rates, we present the growth dynamics of SAM as the sink growth.

Under the constant photoperiod conditions, plant growth was simulated over 10 days at various photoperiods $(8,9,10$, $\ldots, 16 \mathrm{~h})$. We then compared the growth increment over 10 days among plant types by calculating the differences for the following pairs: (wild type-mutant), (homeostatic-mutant), and (homeostatic-wild type). Under the changing photoperiod conditions, plants grown for 5 days in an 8 -h or 16 -h photoperiod were transferred to a 16 -h or 8 -h photoperiod and grown for an additional 5 days (Seki et al., 2017). For the wild type and sugar-insensitive mutant, we mainly analyzed the plants with the subjective dusk $\phi^{*}$ of $10 \mathrm{~h}$. In addition, we also simulated 10-day growth of the plants with various values of $\phi^{*}(8,9,11,12 \mathrm{~h})$ in constant photoperiods to investigate the influence of $\phi^{*}$ on growth dynamics. Other parameter values are listed in Table S1. Numerical integration of the ordinary differential equations was performed with the fourth-order Runge-Kutta method using Mathematica (version 10; Wolfram Research).

\section{RESULTS}

When the plants are grown in a constant photoperiod, the growth of the sink (SAM or RAM) of the all three types increases as the photoperiod is lengthened (Figure 3A). The sugar-sensitive
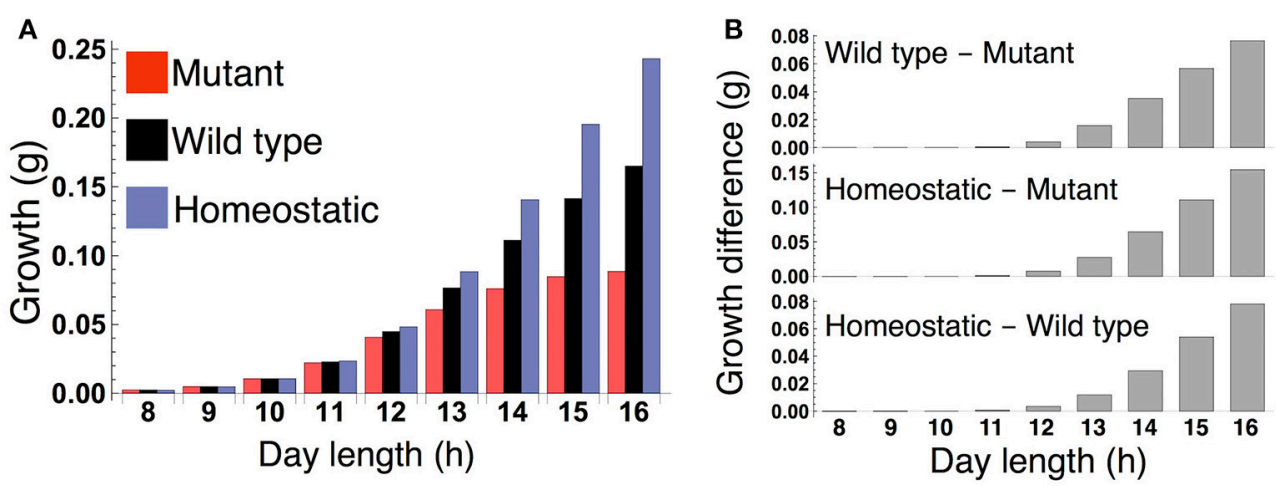

FIGURE 3 | (A) Growth of the sink (SAM or RAM) of the mutant, wild type, and homeostatic plant over 10 days under constant photoperiod conditions. (B) Growth difference between the wild type and mutant (upper), between the homeostatic and mutant (middle), and between the homeostatic and wild type (lower). 
wild type and homeostatic plant grow significantly faster than the sugar-insensitive mutant under long days (Figure 3B). When photoperiod is shorter than $12 \mathrm{~h}$, the growth difference between the mutant and the others almost disappears. Growth of the wild type is slower than that of the homeostatic plant in long photoperiods (Figure 3B), suggesting that the minimization of sucrose fluctuation is the most effective strategy for efficient growth under these conditions. We confirmed the robustness of our results under different parameter values for carbon metabolism (Figure S2) as well as for the phloem tube network (Figure S3).

Under a long day (16 L/ $8 \mathrm{D})$, sucrose levels in the source and sink (SAM or RAM) are highly variable in the sugar-insensitive mutant, moderately variable in the wild type, and almost constant in the homeostatic plant (Figures 4A,B). Diel patterns of growth reflect sucrose profiles in the sink, revealing a substantially lower growth rate during the evening in the mutant and a moderately lower growth rate during the evening in the wild type compared to the homeostatic plant (Figure 4C). The difference in evening growth rate is the major reason for differential growth among the three plant types in a long photoperiod. The mutant accumulates the largest amount of starch, with some amount unused even at the end of night (Figure 4D), indicating inefficient translocation of carbon. In contrast, the wild type accumulates less starch than the mutant and invests a larger amount of photoassimilate for growth (Figure 4D).

The different sucrose, starch, and growth profiles between the wild type and sugar-insensitive mutant are caused by the clock plasticity in response to sugar. The decrease in sucrose levels at dawn (Figure 4A) is sensed by the wild type as a negative sugar signal, driving a phase delay of the circadian oscillator (Figure 4E). This phase delay in the morning increases the starch degradation rate during the light period (Figure 4F), resulting in elevation of the sucrose level (Figure 4A) and decrease of the starch level (Figure 4D). Such a phase shift in the circadian oscillator does not occur in the mutant (Figure 4E). Excessively high sucrose in the mutant around dawn (Figure 4B) does not significantly contribute to growth (Figure 4C) due to the saturating property of the growthrelated sucrose consumption rate $\alpha$ (Figure 2A), while the sucrose decrease around dusk substantially reduces growth rate since at this low level $\alpha$ is almost linearly dependent on sucrose.

Under a short day (8 L/16 D; Figure 5), the wild type and sugar-insensitive mutant show the similar sugar and growth dynamics as reported previously (Seki et al., 2017). The elevation
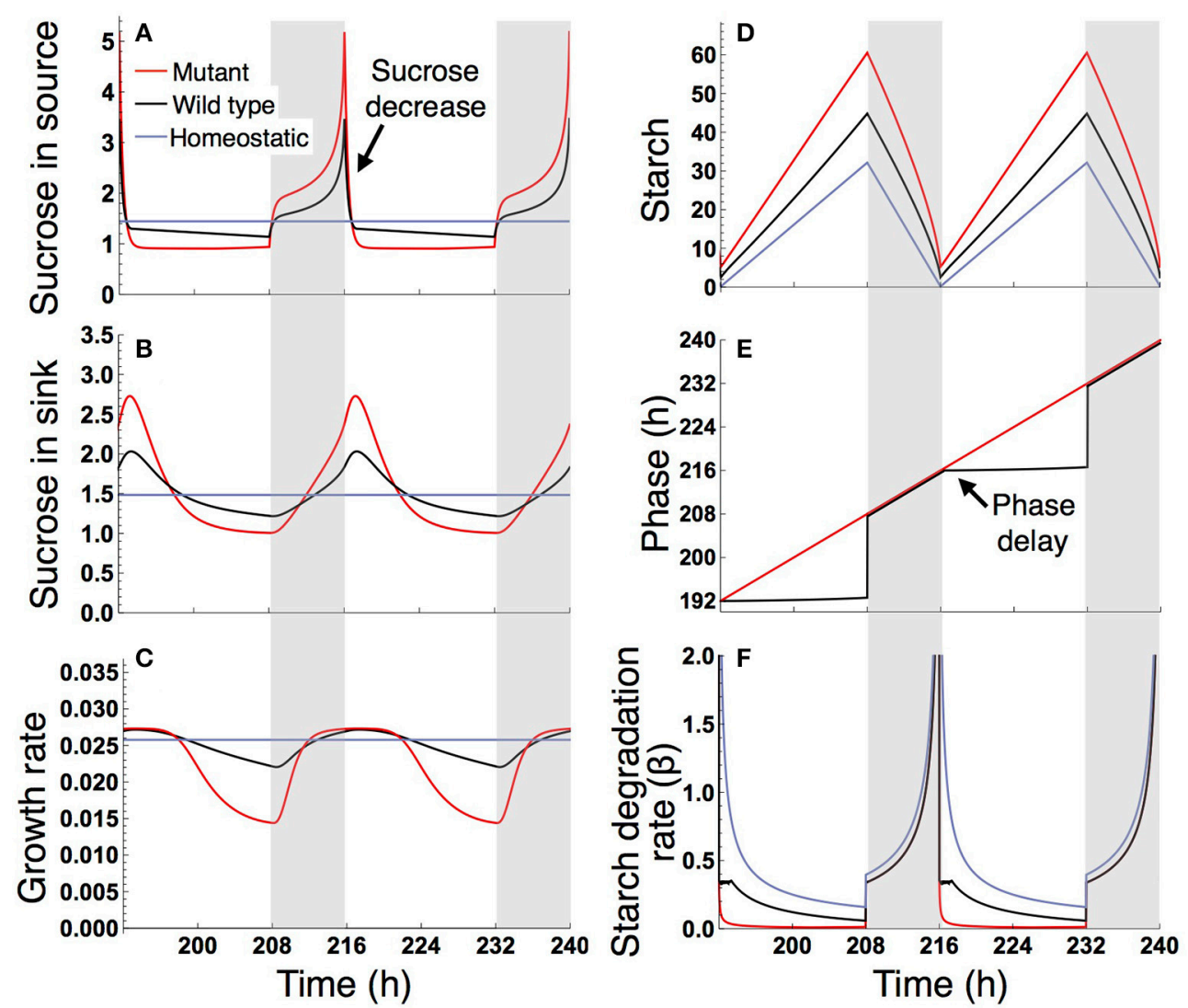

FIGURE 4 | Predicted profiles of (A) sucrose in the source, (B) sucrose in the sink (SAM or RAM), (C) growth rate of the sink (SAM or RAM), (D) starch, (E) phase, and $(\mathbf{F})$ starch degradation rate of the plants grown in a 16-h photoperiod. The unit for sucrose and starch is $\mu \mathrm{mol}_{6} \mathrm{~g}^{-1} \mathrm{FW}$. White background, light period; Gray background, dark period. 

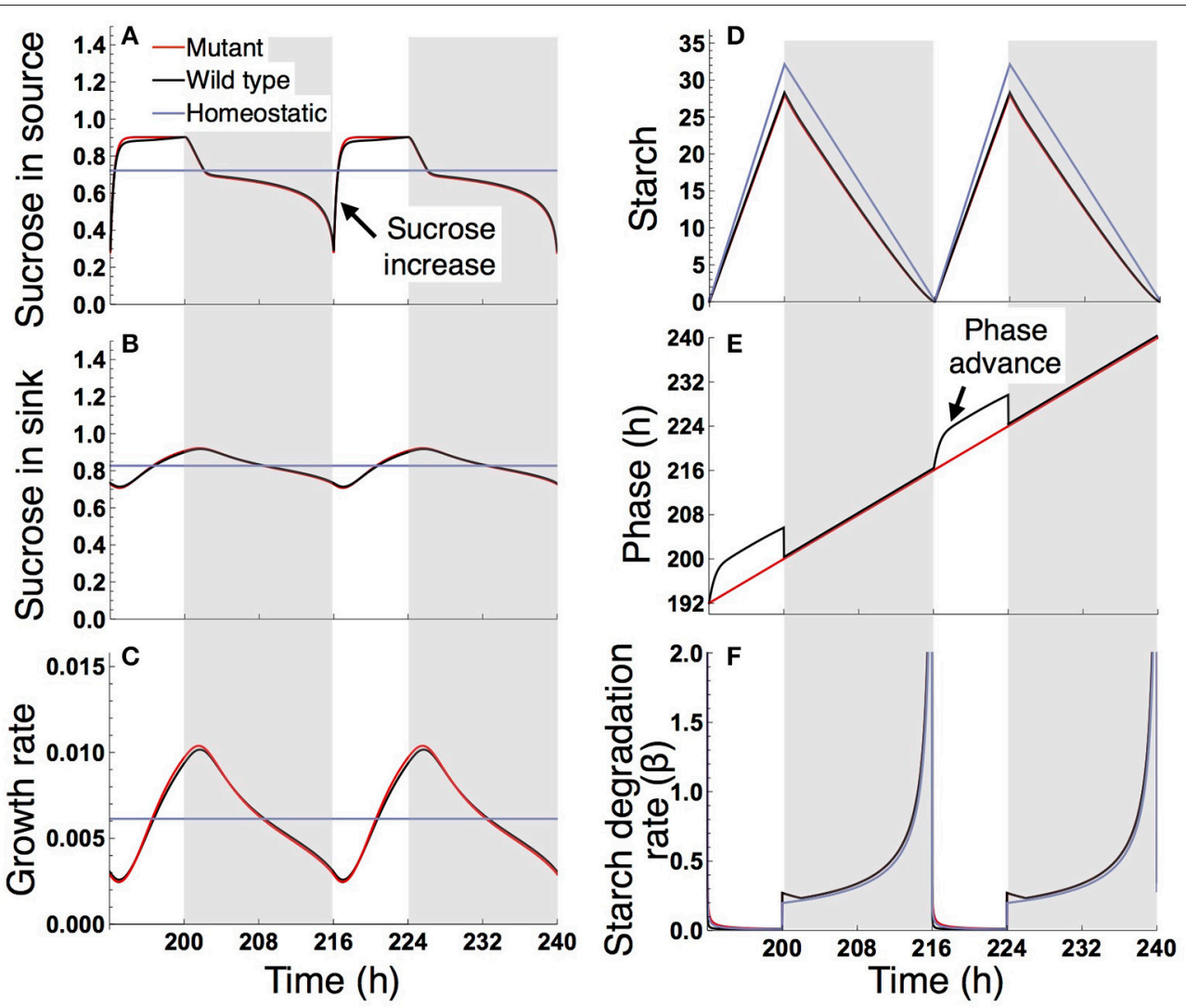

FIGURE 5 | Predicted profiles of (A) sucrose in the source, (B) sucrose in the sink (SAM or RAM), (C) growth rate of the sink (SAM or RAM), (D) starch, (E) phase, and $\mathbf{( F )}$ starch degradation rate of the plants grown in an 8-h photoperiod. Details are as in the legend to Figure $\mathbf{4}$.

of sucrose at dawn (Figure 5A) gives rise to a positive sugar signal and resultant phase advance in the wild type (Figure 5E). However, this phase shift does not substantially change the starch degradation rate (Figure 5F), so the sugar and growth dynamics are almost the same for the wild type and mutant (Figures 5A-D). The homeostatic plant displays distinct growth dynamics (Figure 5C). Nevertheless, the difference in 10-day growth among plants is very small in the short photoperiod (Figure 3B). Since all three plants fix less carbon per day compared to under long day conditions, sucrose concentration is low both in the source and sink (Figures 5A,B). This leads to a slow growth rate due to low supply of sucrose. Because all three plants are limited by sucrose under short day conditions, the growth difference among them is indistinguishably small.

We examined the effect of the timing of the subjective dusk $\phi^{*}$ on growth dynamics of the wild type (Figures 6, 7). Changes in $\phi^{*}$ do not markedly alter the values of 10-day growth of the sink and its difference among plant types (Figure 6). On the other hand, growth rate is strongly dependent on the timing of both the internal and external dusk. When the external dusk occurs later than the internal dusk (i.e., the length of the light period $\tau_{\mathrm{L}}$ is larger than $\left.\phi^{*}\right)$, the growth rate peaks around dawn and decreases around dusk (Figures 4C, 7). As the photoperiod is shortened, amplitude of growth rate decreases and eventually becomes almost zero. When the value of $\tau_{\mathrm{L}}$ is smaller than $\phi^{*}$, the opposite growth pattern is observed, with a peak around dusk and a trough around dawn (Figures 5C, 7).

When the plants are transferred from long to short or short to long days, the growth of the wild type and homeostatic plant are consistently higher than that of the sugar-insensitive mutant (Figure 8A). All three plants are able to restore normal sugar and growth dynamics in about 2 days after the transfer, although the dynamics are complex immediately after the photoperiod change (Figure 8B). Therefore, the growth differences under these conditions may reflect the results under the constant photoperiod conditions that growth of the mutant is inferior to the others especially under a long day (Figure 3). Note that the dynamics of other variables such as sucrose in source were reported in a previous study (Seki et al., 2017).

\section{DISCUSSION}

Our results provide the first theoretical evidence that clock entrainment by photosynthetic products improves the fitness of higher plants. The sugar-sensitive wild type is predicted to grow faster than the sugar-insensitive mutant under long day conditions (Figure 3) because the phase shift of the circadian oscillator by sugar signals enables efficient sugar allocation for growth, while the mutant accumulates carbon 


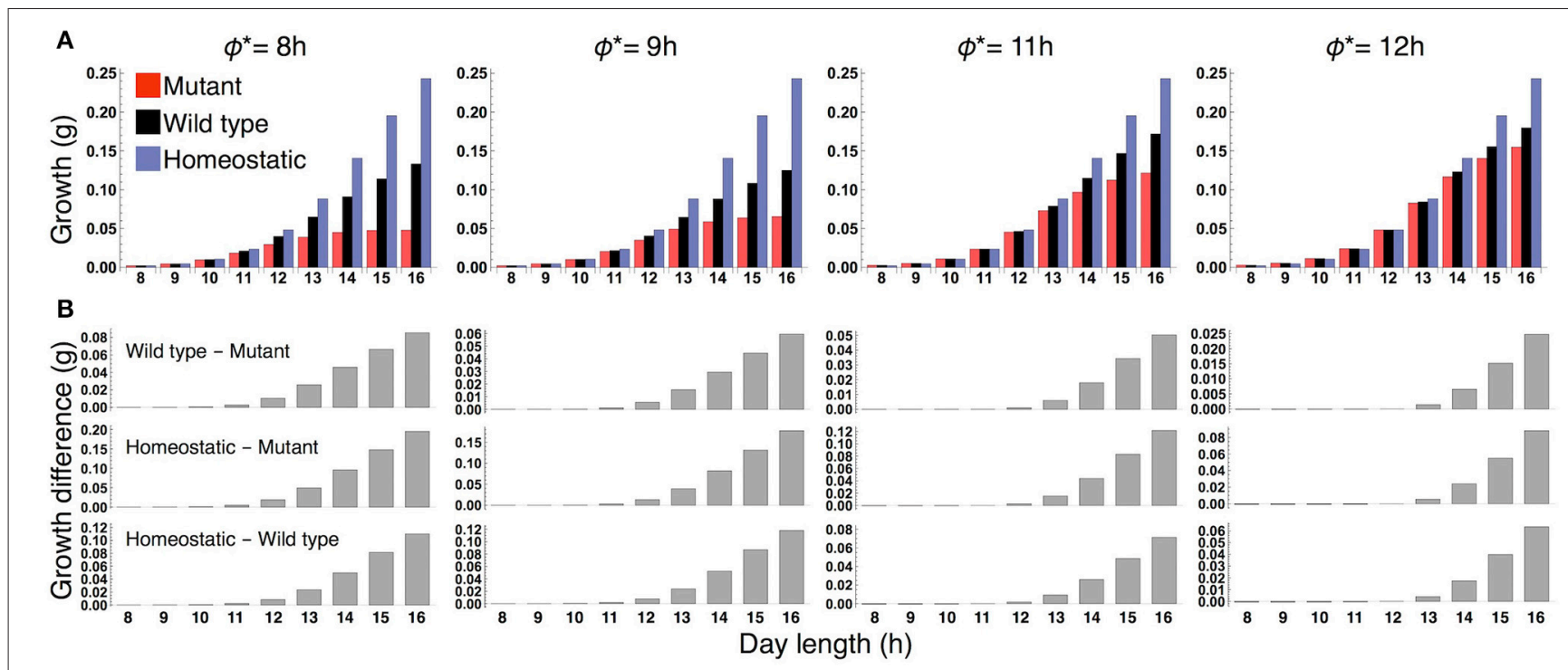

FIGURE 6 | (A) 10-day growth of the sink (SAM or RAM) of the mutant, wild type, and homeostatic plant, and (B) growth differences among the plants at various values of the subjective dusk $\phi^{*}$.

as insoluble starch unusable for growth. The growth of the wild type is also higher than the mutant under changing photoperiod conditions (Figure 8A). Since the wild type and mutant are assumed to possess the same entrainment property to light, the lower growth in the mutant stems solely from the lack of sugar-induced phase adjustment. These results suggest that clock entrainment by endogenous sugar signals, in addition to entrainment by exogenous light signals, optimizes plant growth in nature, where the day length gradually changes.

Our results also provide important information about the internal timing of plants. Our model predicts that the growth pattern of the wild type displays a maximum around dawn and a minimum around dusk in a 16 -h photoperiod (Figure 4C) or more generally under conditions where the value of $\tau_{\mathrm{L}}$ (length of the light period) is larger than $\phi^{*}$ (timing of the subjective dusk) (Figure 7). When the value of $\tau_{\mathrm{L}}$ is smaller than $\phi^{*}$, the pattern is reversed (Figure 5C where $\tau_{\mathrm{L}}=8 \mathrm{~h}$ and $\phi^{*}=10 \mathrm{~h}$; Figure 7). Yazdanbakhsh et al. (2011) reported, in both 16-h and 8 -h photoperiods, a similar diel pattern in root elongation growth of $A$. thaliana (Col-0), which possibly correlates with fresh biomass change since both growth measures consider dry matter production and water content. These findings imply that $\phi^{*}$ of this accession is about $8 \mathrm{~h}$. This idea is consistent with the PRC of the same accession to a sucrose pulse showing a transition from phase advance to delay, which corresponds to the phase $\phi^{*}$, at $6-10 \mathrm{~h}$ zeitgeber time (Haydon et al., 2013; Seki et al., 2017). These data suggest that a relatively short light period is subjectively anticipated by this accession. Other ecotypes of $A$. thaliana that anticipate later subjective dusk (i.e., large $\phi^{*}$ ) will display the inversed growth pattern in a short photoperiod and later phase advance-to-delay transition of the sucrose-pulsed PRC.
Numerous mathematical models on growth of various plants have been developed (e.g., Thornley and Johnson, 1990; Chew et al., 2014, 2017; Feller et al., 2015; Barillot et al., 2016a,b). Among them, two multiscale models of $A$. thaliana include the circadian clock sub-model and are able to quantitatively predict growth (Chew et al., 2014, 2017). Because the phase of the clock is dynamically regulated by sugar signals in our model and such phase adjustment is not considered in the previous models (Chew et al., 2014, 2017), only our model enables us to evaluate the impact of phase regulation by sugar on plant growth. Our model correctly predicts the empirical finding that wild type $A$. thaliana (Col-0) and the mutant prr7-11 in which phase response to sugar is abolished grow similarly in a 12 -h photoperiod (Chew et al., 2017; Figure 3). We suggest that this is due to the relatively weak impact of sugar entrainment on growth, as our modeling results show that the sugar-induced phase shift has less effect on growth dynamics in shorter photoperiods (Figure 5). These considerations are reminiscent of the empirical finding that response of $A$. thaliana to induced increases in trehalose-6phosphate, a potential signal metabolite of sucrose status, was more intense in a $16-\mathrm{h}$ photoperiod than in a 12 -h photoperiod (Figueroa et al., 2016). Our model also predicts significantly faster growth of the wild type than the sugar-insensitive mutant under long day conditions (Figure 3), which will be experimentally tested using wild type $A$. thaliana and prr7-11. Wild type is likely to grow faster than prr7-11 under long day conditions because the latter accumulates more starch than the former in a 16-h photoperiod (Seki et al., 2017) which implies that prr711 can utilize less sucrose for growth than wild type. However, it should be noted that mutation of PRR7 affects not only sugar signaling but also light signaling in the plant circadian system (Kaczorowski and Quail, 2003; Farré et al., 2005), and thus the growth characteristics of prr7-11 can be influenced by 

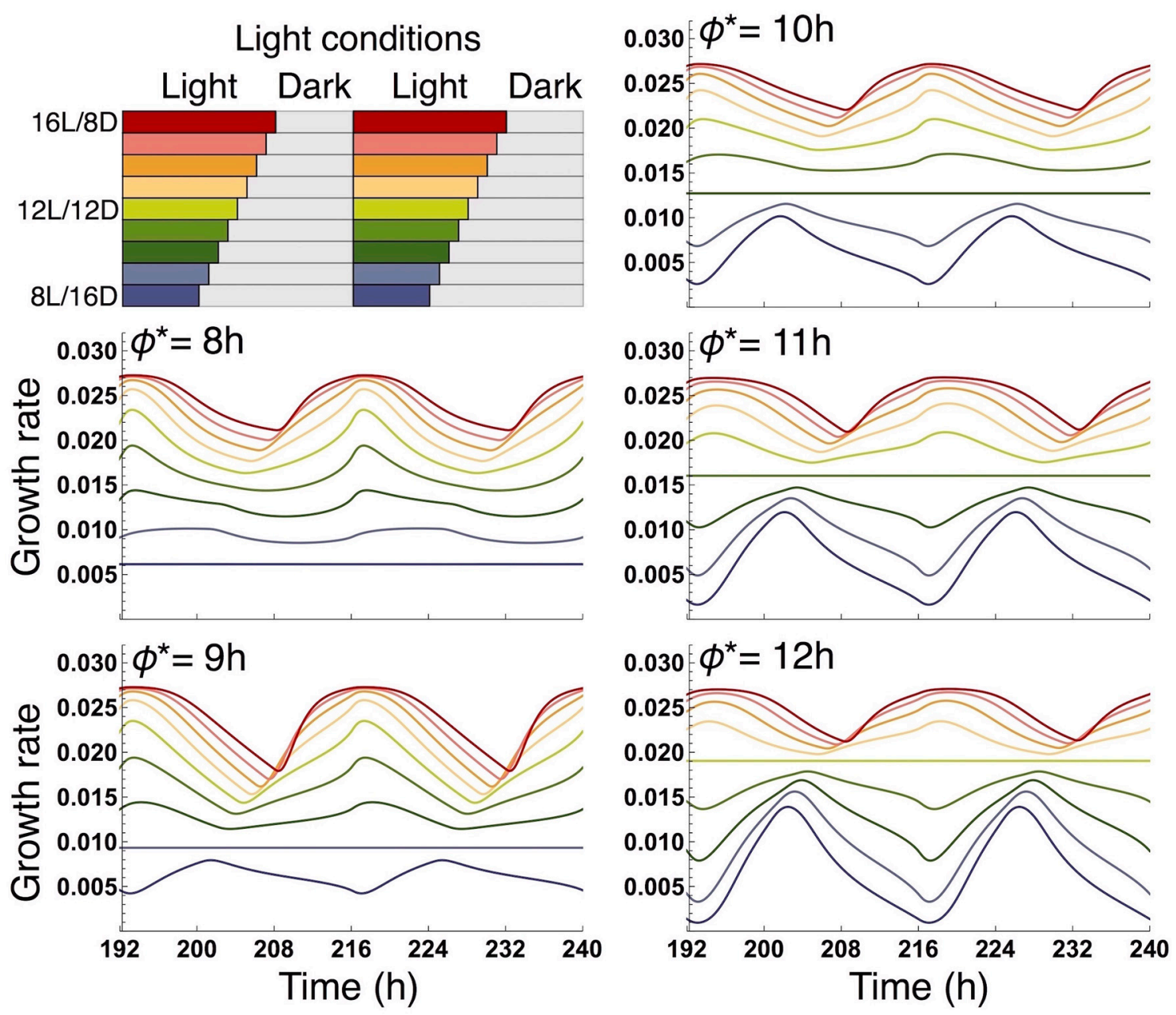

FIGURE 7 | Comparison of growth rate of the sink (SAM or RAM) of the wild type at various values of the subjective dusk $\phi^{\star}$. In each panel, growth dynamics in various photoperiods is represented. Different colors correspond to different light conditions (upper left panel).

variations in light as well as sugar entrainment. Nevertheless, sugar entrainment is likely to be a dominant factor because our previous study has theoretically shown that the deficient in sugar signaling only is sufficient to correctly predict patterns of starch turnover of prr7-11 (Seki et al., 2017).

There are several possibilities to expand our model. We used a constant rate $a$ for carbon capture, which was determined in a previous study based on experiments where nutrient levels were controlled (Gibon et al., 2004; Feugier and Satake, 2013). In higher plants, however, it has been demonstrated that sugar accumulation downregulates photosynthesis, possibly through decreased activity of Rubisco (Araya et al., 2006; Ribeiro et al., 2012; Quentin et al., 2013; Lobo et al., 2015). Formalizing the carbon capture rate as a decreasing function of sucrose concentration could reduce the growth of the sugarinsensitive mutant under long day conditions due to the very high sucrose level around dawn (Figures 4A,B). Regarding circadian clock properties, we considered a circadian oscillator with a period of $24 \mathrm{~h}$ (i.e., $\omega=1$ ). A recent study has reported a positive correlation between the free-running period of natural populations of Mimulus guttatus and the latitude of their geographic origin (Greenham et al., 2017). In A. thaliana, a similar but weak correlation has been found (Michael et al., 2003), although advantages of such variation in the period remain elusive. Growth simulations of plants with fast- and slow-running clocks could potentially reveal the adaptive value of natural variation in the circadian period in terms of growth optimization. Moreover, we can consider the possibility that two or more clock genes are involved in the phase response to sugar, where each gene can have differential responsiveness. Although the crucial role of PRR7 in the response has been established (Haydon et al., 2013), other clock genes could participate in it; for instance, CCA1 will be a good candidate, mutation of which disrupts the dependency of the circadian period on sucrose concentration in growth media (Haydon et al., 2013). To examine this possibility, the single phase oscillator will be expanded to coupled two or multiple oscillators model, in which each phase oscillator is defined with different PRC to sucrose and sugar input function 

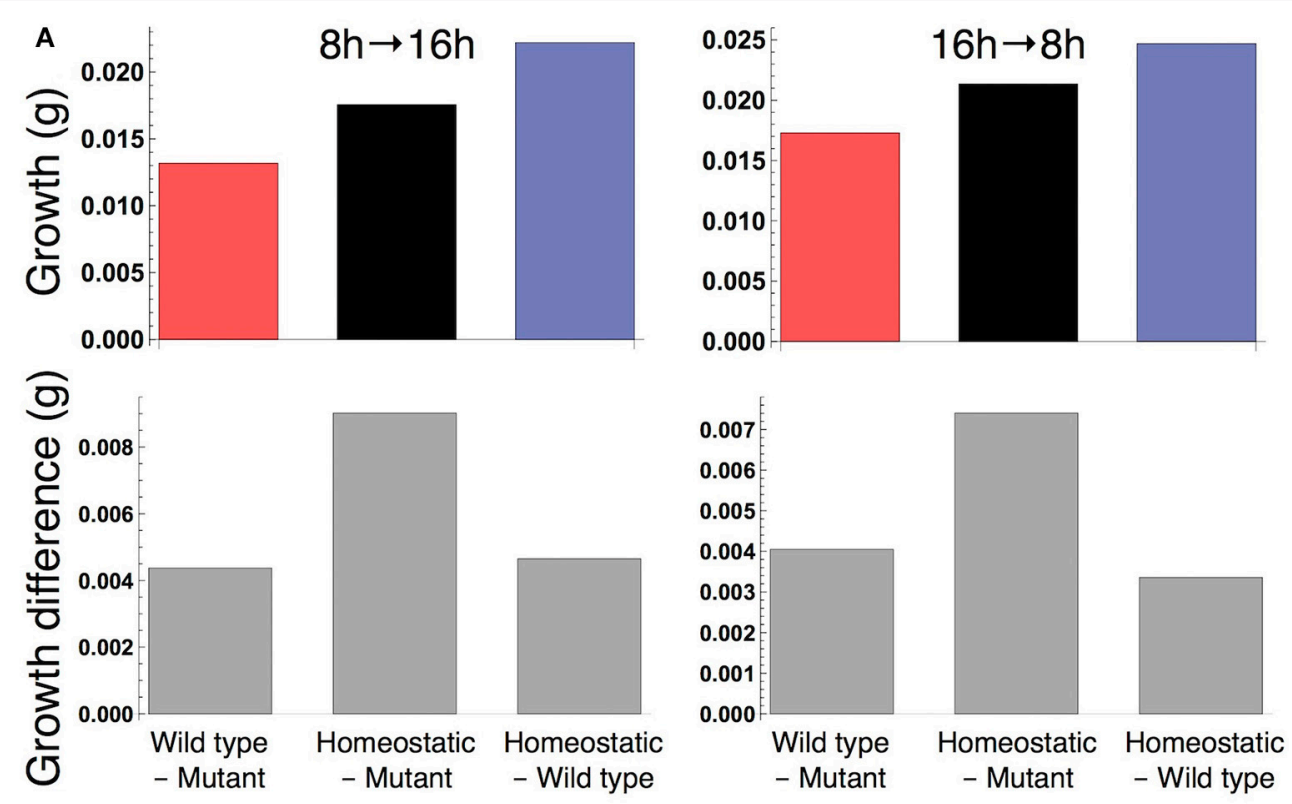

B
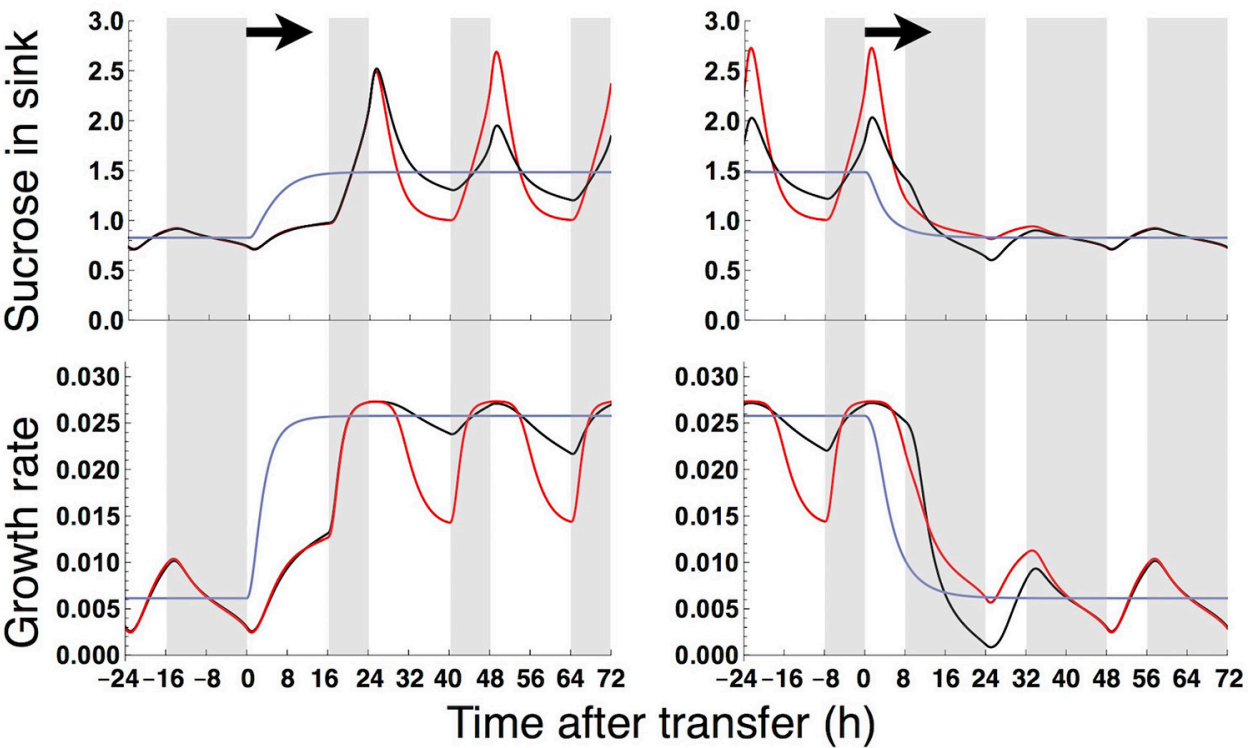

FIGURE 8 | (A) Growth of the sink (SAM or RAM) of the mutant, wild type, and homeostatic plant transferred from an 8-h to 16-h photoperiod (left) and from a 16-h to 8-h photoperiod (right) and the growth difference among the plants. Color codes as in Figure $\mathbf{3 A}$. (B) Predicted profiles of sucrose in the sink and growth rate around the photoperiod change. Details are as in the legend to Figure 4.

( $Z_{\mathrm{S}}$ and $f_{\mathrm{S}}$ in Equation (4), respectively). Alternative way is to use the clock gene-regulatory network model (Fogelmark and Troein, 2014; De Caluwé et al., 2016) with the explicit formalization of the interaction between clock genes and sugar signals.

We conclude that plants optimize growth by monitoring nutrient status and utilizing endogenous sugar signals as circadian-phase regulators. Photosynthetic products act not only as direct growth substances but also as feedback signals to the clock to realize the efficient carbon-usage for growth. It is plausible that plants as sessile organisms utilize signals from metabolism because they seem more controllable than environmental signals. In this sense, the phase regulation by endogenous signals is potentially even more important for plants than animals that also utilize metabolic signals as zeitgebers (Woller et al., 2016). Our data strongly support the concept that the circadian clock improves the fitness of organisms by forming complex feedback loops with the signaling pathways it controls (Sanchez and Kay, 2016). 


\section{AUTHOR CONTRIBUTIONS}

TO and AS developed the model and wrote the manuscript. TO analyzed the model.

\section{FUNDING}

TO was supported by Grant-in-Aid for JSPS Fellows Number 16J04492. AS was supported by JSPS KAKENHI Number BR150301.

\section{REFERENCES}

Araya, T., Noguchi, K., and Terashima, I. (2006). Effects of carbohydrate accumulation on photosynthesis differ between sink and source leaves of Phaseolus vulgaris L. Plant Cell Physiol. 47, 644-652. doi: 10.1093/pcp/pcj033

Baena-González, E., and Sheen, J. (2008). Convergent energy and stress signaling. Trends Plant Sci. 13, 474-482. doi: 10.1016/j.tplants.2008.06.006

Barillot, R., Chambon, C., and Andrieu, B. (2016a). CN-Wheat, a functional-structural model of carbon and nitrogen metabolism in wheat culms after anthesis. I. Model description. Ann. Bot. 118, 997-1013. doi: $10.1093 / \mathrm{aob} / \mathrm{mcw} 143$

Barillot, R., Chambon, C., and Andrieu, B. (2016b). CN-Wheat, a functional-structural model of carbon and nitrogen metabolism in wheat culms after anthesis. II. Model evaluation. Ann. Bot. 118, 1015-1031. doi: $10.1093 / \mathrm{aob} / \mathrm{mcw} 144$

Caspar, T., Huber, S. C., and Somerville, C. (1985). Alterations in growth, photosynthesis, and respiration in a starchless mutant of Arabidopsis thaliana (L.) deficient in chloroplast phosphoglucomutase activity. Plant Physiol. 79, 11-17. doi: 10.1104/pp.79.1.11

Chew, Y. H., Seaton, D. D., Mengin, V., Flis, A., Mugford, S. T., Smith, A. M., et al. (2017). Linking circadian time to growth rate quantitatively via carbon metabolism. Biorxiv doi: 10.1101/105437

Chew, Y. H., Wenden, B., Flis, A., Mengin, V., Taylor, J., Davey, C. L., et al. (2014). Multiscale digital Arabidopsis predicts individual organ and whole-organism growth. Proc. Natl. Acad. Sci. U.S.A. 111, 4127-4136. doi: 10.1073/pnas.1410238111

Christophe, A., Letort, V., Hummel, I., Coumède, P.-H., de Reffye, P., and Lecoeur, J. (2008). A model-based analysis of the dynamics of carbon balance at the whole-plant level in Arabidopsis thaliana. Funct. Plant Biol. 35, 1147-1162. doi: 10.1071/FP08099

De Caluwé, J., Xiao, Q., Hermans, C., Verbruggen, N., Leloup, J.-C., and Gonze, D. (2016). A compact model for the complex plant circadian clock. Front. Plant Sci. 7:74. doi: 10.3389/fpls.2016.00074

Deprost, D., Yao, L., Sormani, R., Moreau, M., Leterreux, G., Nicolai, M., et al. (2007). The Arabidopsis TOR kinase links plant growth, yield, stress resistance and mRNA translation. EMBO Rep. 8, 864-870. doi: 10.1038/sj.embor.7401043

Dodd, A. N., Salathia, N., Hall, A., Kévei, E., Tóth, R., Nagy, F., et al. (2005). Plant circadian clocks increase photosynthesis, growth, survival, and competitive advantage. Science 309, 630-633. doi: 10.1126/science.1115581

Farré, E. M., Harmer, S. L., Harmon, F. G., Yanovsky, M. J., and Kay, S. A. (2005). Overlapping and distinct roles of PRR7 and PRR9 in the Arabidopsis circadian clock. Curr. Biol. 15, 47-54. doi: 10.1016/j.cub.2004.12.067

Feller, C., Favre, P., Janka, A., Zeeman, S. C., Gabriel, J.-P., and Reinhardt, D. (2015). Mathematical modeling of the dynamics of shoot-root interactions and resource partitioning in plant growth. PLoS ONE 10:e0127905. doi: 10.1371/journal.pone.0127905

Feugier, F. G., and Satake, A. (2013). Dynamical feedback between circadian clock and sucrose availability explains adaptive response of starch metabolism to various photoperiods. Front. Plant Sci. 3:305. doi: 10.3389/fpls.2012. 00305

Figueroa, C. M., Feil, R., Ishihara, H., Watanabe, M., Kölling, K., Krause, U., et al. (2016). Trehalose 6-phosphate coordinates organic and amino acid metabolism with carbon availability. Plant J. 85, 410-423. doi: 10.1111/tpj.13114

\section{ACKNOWLEDGMENTS}

We thank Prof. Alex A. R. Webb, Dr. Camila Caldana, and Dr. Motohide Seki for fruitful discussions and useful comments on the manuscript.

\section{SUPPLEMENTARY MATERIAL}

The Supplementary Material for this article can be found online at: https://www.frontiersin.org/articles/10.3389/fpls.2017. 01859/full\#supplementary-material

Fogelmark, K., and Troein, C. (2014). Rethinking transcriptional activation in the Arabidopsis circadian clock. PLoS Comput. Biol. 10:e1003705. doi: 10.1371/journal.pcbi.1003705

Gibon, Y., Bläsing, O. E., Palacios-Rojas, N., Pankovic, D., Hendriks, J. H. M., Fisahn, J., et al. (2004). Adjustment of diurnal starch turnover to short days: depletion of sugar during the night leads to a temporary inhibition of carbohydrate utilization, accumulation of sugars and post-translational activation of ADP-glucose pyrophosphorylase in the following light period. Plant J. 39, 847-862. doi: 10.1111/j.1365-313X.2004.02173.x

Graf, A., and Smith, A. M. (2011). Starch and the clock: the dark side of plant productivity. Trends Plant Sci. 16, 169-175. doi: 10.1016/j.tplants.2010.12.003

Graf, A., Schlereth, A., Stitt, M., and Smith, A. M. (2010). Circadian control of carbohydrate availability for growth in Arabidopsis plants at night. Proc. Natl. Acad. Sci. U.S.A. 107, 9458-9463. doi: 10.1073/pnas.0914299107

Greenham, K., Lou, P., Puzey, J. R., Kumar, G., Arnevik, C., Farid, H., et al. (2017). Geographic variation of plant circadian clock function in natural and agricultural settings. J. Biol. Rhythms 32, 26-34. doi: $10.1177 / 0748730416679307$

Haydon, M. J., Mielczarek, O., Robertson, F. C., Hubbard, K. E., and Webb, A. A. R. (2013). Photosynthetic entrainment of the Arabidopsis thaliana circadian clock. Nature 502, 689-692. doi: 10.1038/nature12603

Johnson, C. H., Elliott, J. A., and Foster, R. (2003). Entrainment of circadian programs. Chronobiol. Int. 20, 741-774. doi: 10.1081/CBI-120024211

Kaczorowski, K. A., and Quail, P. H. (2003). Arabidopsis pseudo-response regulator7 is a signaling intermediate in phytochrome-regulated seedling deetiolation and phasing of the circadian clock. Plant Cell 15, 2654-2665. doi: 10.1105/tpc.015065

Lastdrager, J., Hanson, J., and Smeekens, S. (2014). Sugar signals and the control of plant growth and development. J. Exp. Bot. 65, 799-807. doi: 10.1093/jxb/ert474

Lobo, A. K. M., Martins, M. O., Neto, M. C. L., Machado, E. C., Ribeiro, R. V., and Silveira, J. A. G. (2015). Exogenous sucrose supply changes sugar metabolism and reduces photosynthesis of sugarcane through the downregulation of Rubisco abundance and activity. J. Plant Physiol. 179, 113-121. doi: 10.1016/j.jplph.2015.03.007

Locke, J. C. W., Southern, M. M., Kozma-Bognár, L., Hibberd, V., Brown, P. E., Turner, M. S., et al. (2005). Extension of a genetic network model by iterative experimentation and mathematical analysis. Mol. Syst. Biol. 1:0013. doi: $10.1038 / \mathrm{msb} 4100018$

Lu, Y., Gehan, J. P., and Sharkey, T. D. (2005). Daylength and circadian effects on starch degradation and maltose metabolism. Plant Physiol. 138, 2280-2291. doi: 10.1104/pp.105.061903

Mengin, V., Pyl, E.-T., Moraes, T. A., Sulpice, R., Krohn, N., Encke, B., et al. (2017). Photosynthate partitioning to starch in Arabidopsis thaliana is insensitive to light intensity but sensitive to photoperiod due to a restriction on growth in the light in short photoperiods. Plant Cell Environ. 40, 2608-2627. doi: $10.1111 /$ pce. 13000

Michael, T. P., Salomé, P. A., Yu, H. J., Spencer, T. R., Sharp, E. L., McPeek, M. A., et al. (2003). Enhanced fitness conferred by naturally occurring variation in the circadian clock. Science 302, 1049-1053. doi: 10.1126/science. 1082971

Münch, E. (1930). Die Stoffbewegung in der Pflanze. Jena: Fischer.

Quentin, A. G., Close, D. C., Hennen, L. M. H. P., and Pinkard, E. A. (2013). Down-regulation of photosynthesis following girdling, but contrasting effects 
on fruit set and retention, in two sweet cherry cultivars. Plant Physiol. Biochem. 73, 359-367. doi: 10.1016/j.plaphy.2013.10.014

Ribeiro, R. V., Machado, E. C., Habermann, G., Santos, M. G., and Oliveira, R. F. (2012). Seasonal effects on the relationship between photosynthesis and leaf carbohydrates in orange trees. Funct. Plant Biol. 39, 471-480. doi: 10.1071/FP11277

Sanchez, S. E., and Kay, S. A. (2016). The plant circadian clock: from a simple timekeeper to a complex developmental manager. Cold Spring Harbor Perspect. Biol. 8:a027748. doi: 10.1101/cshperspect.a027748

Satake, A., Seki, M., Iima, M., Teramoto, T., and Nishiura, Y. (2016). Florigen distribution determined by a source-sink balance explains the diversity of inflorescence structures in Arabidopsis. J. Theor. Biol. 395, 227-237. doi: 10.1016/j.jtbi.2016.01.035

Scialdone, A., Mugford, S. T., Feike, D., Skeffington, A., Borrill, P., Graf, A., et al. (2013). Arabidopsis plants perform arithmetic division to prevent starvation at night. Elife 2:e00669. doi: 10.7554/eLife.00669

Seki, M., Feugier, F. G., Song, X.-J., Ashikari, M., Nakamura, H., Ishiyama, K., et al. (2015). A mathematical model of phloem sucrose transport as a new tool for designing rice panicle structure for high grain yield. Plant Cell Physiol. 56, 605-619. doi: 10.1093/pcp/pcu191

Seki, M., Ohara, T., Hearn, T. J., Frank, A., da Silva, V. C. H., Caldana, C., et al. (2017). Adjustment of the Arabidopsis circadian oscillator by sugar signaling dictates the regulation of starch metabolism. Sci. Rep. 7:8305. doi: 10.1038/s41598-017-08325-y

Smith, A. M., and Stitt, M. (2007). Coordination of carbon supply and plant growth. Plant Cell Environ. 30, 1126-1149. doi: 10.1111/j.1365-3040.2007. 01708.x

Smith, S. M., Fulton, D. C., Chia, T., Thorneycroft, D., Chapple, A., Dunstan, H., et al. (2004). Diurnal changes in the transcriptome encoding enzymes of starch metabolism provide evidence for both transcriptional and posttranscriptional regulation of starch metabolism in Arabidopsis leaves. Plant Physiol. 136, 2687-2699. doi: $10.1104 /$ pp.104.044347
Stitt, M., and Zeeman, S. C. (2012). Starch turnover: pathways, regulation and role in growth. Curr. Opin. Plant Biol. 15, 282-292. doi: 10.1016/j.pbi.2012.03.016

Sulpice, R., Flis, A., Ivakov, A. A., Apelt, F., Krohn, N., Encke, B., et al. (2014). Arabidopsis coordinates the diurnal regulation of carbon allocation and growth across a wide range of photoperiods. Mol. Plant 7, 137-155. doi: $10.1093 / \mathrm{mp} / \mathrm{sst} 127$

Thornley, J. H. M., and Johnson, I. R. (1990). Plant and Crop Modeling: A Mathematical Approach to Plant and Crop Physiology. Oxford: Clarendon Press.

Woelfle, M. A., Ouyang, Y., Phanvijhitsiri, K., and Johnson, C. H. (2004). The adaptive value of circadian clocks: an experimental assessment in cyanobacteria. Curr. Biol. 14, 1481-1486. doi: 10.1016/j.cub.2004.08.023

Woller, A., Duez, H., Staels, B., and Lefranc, M. (2016). A mathematical model of the liver circadian clock linking feeding and fasting cycles to clock function. Cell Rep. 17, 1087-1097. doi: 10.1016/j.celrep.2016.09.060

Yazdanbakhsh, N., Sulpice, R., Graf, A., Stitt, M., and Fisahn, J. (2011). Circadian control of root elongation and $\mathrm{C}$ partitioning in Arabidopsis thaliana. Plant Cell Environ. 34, 877-894. doi: 10.1111/j.1365-3040.2011. 02286.x

Younginger, B. S., Sirová, D., Cruzan, M. B., and Ballhorn, D. J. (2017). Is biomass a reliable estimate of plant fitness? Appl. Plant Sci. 5:1600094. doi: 10.3732/apps.1600094

Conflict of Interest Statement: The authors declare that the research was conducted in the absence of any commercial or financial relationships that could be construed as a potential conflict of interest.

Copyright $\odot 2017$ Ohara and Satake. This is an open-access article distributed under the terms of the Creative Commons Attribution License (CC BY). The use, distribution or reproduction in other forums is permitted, provided the original author(s) or licensor are credited and that the original publication in this journal is cited, in accordance with accepted academic practice. No use, distribution or reproduction is permitted which does not comply with these terms. 